\title{
Medical dispatchers recognise substantial amount of acute stroke during emergency calls
}

Søren Viereck ${ }^{1 *}$ (D) Thea Palsgaard Møller ${ }^{1}$, Helle Klingenberg Iversen ${ }^{2}$, Hanne Christensen $^{3}$ and Freddy Lippert ${ }^{1}$

\begin{abstract}
Background: Immediate recognition of stroke symptoms is crucial to ensure timely access to revascularisation therapy. Medical dispatchers ensure fast admission to stroke facilities by prioritising the appropriate medical response. Data on medical dispatchers' ability to recognise symptoms of acute stroke are therefore critical in organising emergency stroke care.

We aimed to describe the sensitivity and positive predictive value of medical dispatchers' ability to recognise acute stroke during emergency calls, and to identify factors associated with recognition.

Methods: This was an observational study of 2653 consecutive unselected patients with a final diagnosis of stroke or transient ischemic attack (TIA). All admitted through the Emergency Medical Services Copenhagen, during a 2-year study period (2012-2014). Final diagnoses were matched with dispatch codes from the Emergency Medical Dispatch Centre. Sensitivity and positive predictive value were calculated. The effect of age, gender, and time-of-day was analysed using multivariable logistic regression.
\end{abstract}

Results: The sensitivity was $66.2 \%$ (95 \% Cl: $64.4 \%-68.0 \%$ ), and the positive predictive value was $30.2 \%$ (95 \% Cl: $29.1 \%-31.4 \%)$. The multivariable logistic regression analyses showed that emergency calls during daytime and a final diagnosis of TIA vs. intracerebral haemorrhage (ICH), was positively associated with recognition of stroke (OR 2. 70, 95 \% Cl: 2.04-3.57).

Discussion: This study reports a high rate of stroke recognition compared to other studies ranging from $31 \%$ to 74\%. The high sensitivity is likely the result of a profound reorganisation of the Emergency Medical ServicesCopenhagen, including the introduction of EMDs with a medical profession, and a criteria-based dispatch tool. A recognition rate of $100 \%$ is not obtainable without an inappropriate amount of false positive cases.

Conclusions: We report an overall high recognition of stroke by medical dispatchers. A final diagnosis of TIA, compared to $\mathrm{ICH}$, was positively associated with recognition of acute stroke. Emergency medical dispatchers serve as the essential first step in ensuring fast-track stroke treatment, which would promote timely acute therapy.

Trial registration: Unique identifier: NCT02191514.

Keywords: Stroke, Emergency Medical Services, Medical decision making, Emergency Medical Dispatch

\footnotetext{
* Correspondence: viereck.soeren@gmail.com

${ }^{1}$ Emergency Medical Services Copenhagen, University of Copenhagen,

Telegrafvej 5, 2750 Ballerup, Denmark

Full list of author information is available at the end of the article
} 


\section{Background}

Intravenous thrombolysis treatment reduces long-term mortality [1] and is proven efficient and safe up to $4.5 \mathrm{~h}$ after onset of acute ischemic stroke (AIS) [2]. Moreover, patients' morbidity and mortality after AIS is dependent on the time from symptom-onset to definitive care [3-5]. In-hospital time reduction for stroke patients has been an area of great progress. The median time from arrival at the hospital to thrombolysis treatment is reported as short as $21 \mathrm{~min}$ [6].

Despite a safe and effective treatment, the amount of AIS patients receiving thrombolysis ranges between 11 $\%$ and $18 \%$ in Denmark [7]. Excess time from symptomonset to in-hospital evaluation is reported the main reason why physicians refrain from thrombolysis [8].

Time from symptom onset to interventional treatment depends on patient awareness of emergency symptoms and contact to the emergency medical services (EMS) through an emergency call. Transportation by EMS, as opposed to private transport, and pre-arrival notification of the hospital stroke team is associated with shorter prehospital and in-hospital time respectively $[9,10]$. Therefore, provision of the adequate ambulance response dispatched from the Emergency Medical Dispatch Centre (EMDC) is essential.

A vital point in this work flow is recognition of stroke by the Emergency Medical Dispatcher (EMD) [11]. Recognition of stroke is a prerequisite for the necessary prehospital assessment, prehospital notification of the in-hospital stroke team, and appropriate transport to the hospital - ultimately ensuring the best patient care as recommended in Danish and international guidelines $[12,13]$. In order to improve stroke recognition, it is vital to report results from EMDCs around the world and to explore barriers for recognition of stroke. This has the potential to increase the EMDs awareness when handling stroke patients, as well as the future development of dispatch systems.

The aim of this study was two-fold:

1. To determine the sensitivity and the positive predictive value of stroke recognition by EMDs during emergency calls

2. To evaluate if patient's sex, time of day, or final diagnosis was associated with stroke recognition

\section{Methods}

\section{Study design}

We performed an observational register-based study. To determine if the EMD recognised the stroke during the emergency call, we studied dispatch codes from the EMDC. We included patients registered at hospital discharge with a final main diagnosis of acute ischemic stroke (AIS), intracerebral haemorrhage (ICH), or transient ischemic attack (TIA).
This study included TIA, ICH, and AIS but excluded subarachnoid haemorrhage (SAH). The main focus of this study was recognition of symptomatology, in which SAH differs substantially. The term "stroke" will refer to $\mathrm{ICH}$ and AIS throughout the paper.

\section{Setting}

The study was conducted in the Capital Region of Denmark, covering the Copenhagen area and the surrounding suburbs. It inhabits 1.75 million people in an area of $2561 \mathrm{~km}^{2}$.

Thrombolysis treatment is centralised in two stroke centres, which are responsible for the stroke fast track care on alternate days.

\section{EMS setting}

In Denmark, one emergency phone-number (1-1-2) leads to a common call centre that handles police, fire, and medical emergencies. In case of a medical emergency, the caller is forwarded to an EMDC where an EMD handles and prioritises the call. To assist the EMD, an electronic criteria-based decision tool (Danish Index for Emergency Care) [14] is available. All EMDs are either registered nurses or paramedics. They have received 6 weeks of additional training in communication and the use of Danish Index for Emergency Care. The tool suggests a default priority of response ranging from A to F, depending on the caller's main complaint and urgency of the condition. Priority $\mathrm{A}$ is the most acute response and priority $\mathrm{F}$ is non-urgent. The EMDC Copenhagen handles approximately 105,000 emergency calls annually. In case of suspected stroke, the default response is a priority " $\mathrm{A}$ " ambulance manned with either paramedics or emergency medical technicians. Neither the EMDs or the ambulance personnel uses a strict standardised tool, like the Los Angeles Prehospital Stroke Screen [15], for evaluation of stroke symptoms.

\section{Prehospital response setting}

When the ambulance personnel suspects a stroke within the time window for thrombolysis, they consult the stroke neurologist on call at the stroke centre. If the patient is considered eligible for thrombolysis, the patient will be transported to the stroke centre for further examination.

The median response time for patients suitable for thrombolysis (priority A) in Copenhagen is five minutes [16].

\section{Participants}

We used two data sources for identification of stroke patients: The National Patient Registry (NPR) [17] and the Danish Stroke Registry (DSR) [18]. Patients registered with International Classification of Diseases, Tenth Revision (ICD-10) codes, corresponding to TIA (DG459), ICH 


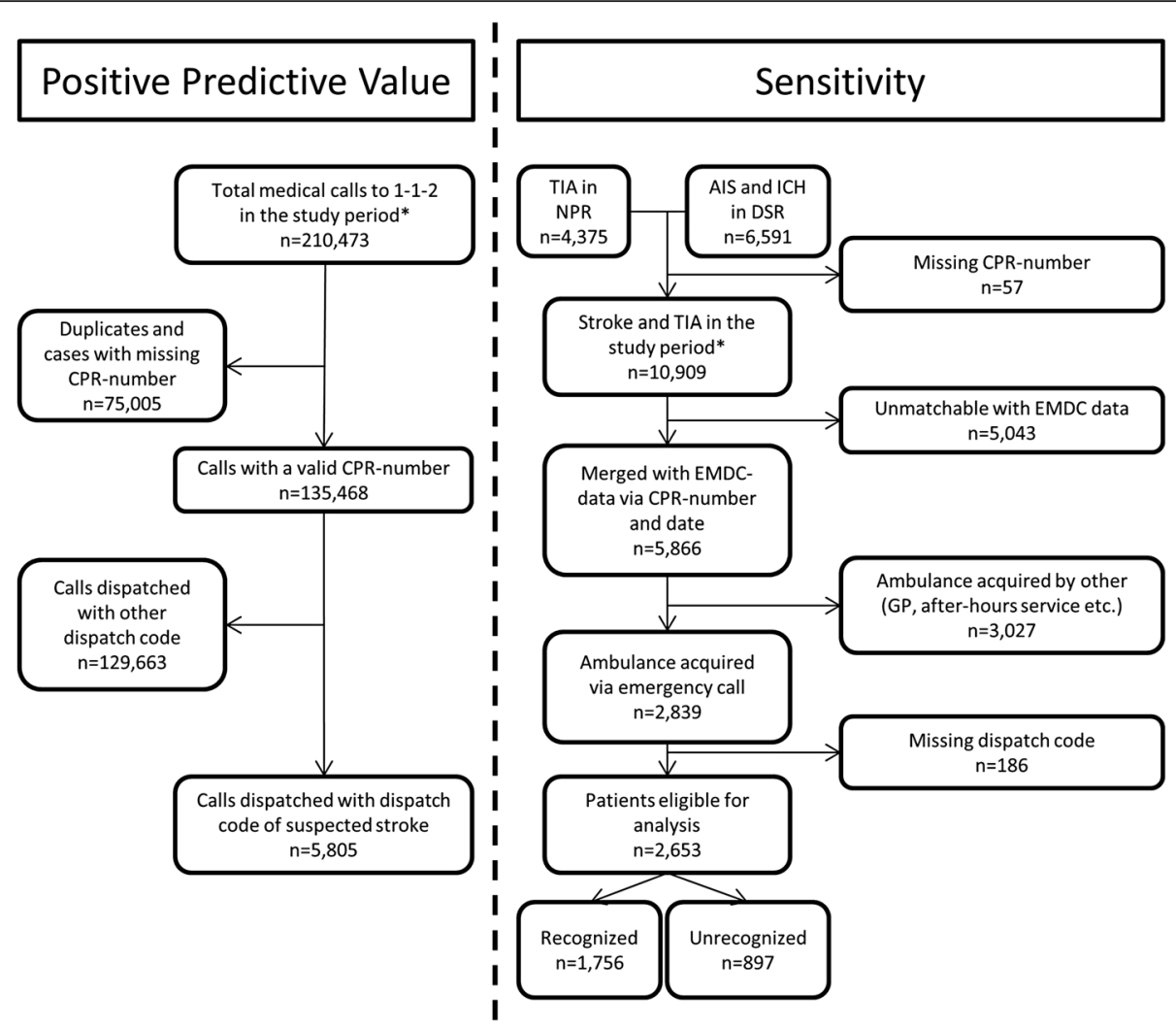

Fig. 1 Flowchart describing the participants divided in the calculation of sensitivity and positive predictive value. *Study Period: $1^{\text {st }}$ of January $2012-$ $31^{\text {st }}$ of December 2013. NPR: National Patient Registry, DSR: Danish Stroke Registry, CPR-number: Unique Personal Identification number, TIA: Transient Ischemic Attack, AIS: Acute Ischemic stroke, ICH: Intracerebral Haemorrhage, GP: General Practitioner, EMDC: Emergency Medical Dispatch Centre

(DI61) or AIS (DI63) in the period from $1^{\text {st }}$ of January 2012 to $31^{\text {st }}$ of December 2013, in the Capital Region of Denmark were included. At the time of study, TIA was not registered in the DSR, therefore data on TIA was collected from the NPR (see Fig. 1). To evaluate if stroke was recognised by the EMD, we identified the patients in the EMDC database using the unique Danish civil registration number (CPR-number) [19] and date of admission as a unique key for data merging. Exclusion criteria were: If CPR-number was missing, if patients were registered in the DSR with a diagnosis of "unspecified stroke (DI64) ", if the case could not be merged with EMDC data, or if patients were transported to the hospital by an ambulance not requested through an emergency call.

\section{Derived variables}

- The following ICD-10 codes were identified in the NPR: DG459 (TIA), and DSR: DI61 (ICH) and DI63 (AIS).

- Sex and age were derived from the CPR-number, which was registered during the emergency call and upon arrival at the hospital. Age was registered as a continuous variable.
- For calculation of diurnal variation, three time intervals were defined: Night (23:00-06:59), day (07:00-14:59), and evening (15:00-22:59).

- Dispatch codes and response priority were obtained from the EMDC database. A dispatch code is assigned for each emergency call, containing information about urgency of the condition and main complaint. The following dispatch codes from the Danish Index for Emergency Care were considered as suspected stroke: A.26.03:" Lowered consciousness-paralysis, suspected stroke: Suddenly asymmetry in the face, lowered force in arms/legs, and/or verbal difficulties" or A.26.04: "Lowered consciousness-paralysis, increasingly unclear/ listless-suspected stroke". All other dispatch codes were defined as "not stroke".

\section{Statistical methods}

To investigate recognition of TIA, ICH, and AIS by EMDs, sensitivity and positive predictive value (PPV) with $95 \%$ confidence intervals $(\mathrm{CI})$ were calculated. To analyse factors associated with recognition of stroke; sensitivity of stroke recognition within final diagnoses, diurnal variations, and sex were compared with Chi2test, and multivariable logistic regression analyses were 
conducted. They were adjusted for confounding variables and after assessment of goodness-of-fit of the model, a scale parameter was added in order to account for over dispersion. Results are presented as absolute numbers, percentages, and odds ratios (OR). Data management and statistical analyses were performed using SAS EG 6.1 statistical software.

This study is part of a larger research project: "Prehospital management of stroke patients by the EMS", which is protocolled online at www.clinicaltrials.gov (NCT02191514). Approval from Danish data protection agency (2007-580015) was obtained. Approval from the regional ethics committee was not needed (H-4-2014-FSP).

\section{Results}

\section{Study population}

We included 10,966 patients in the study: 4375 TIA's and 6591 strokes. Of 2839 patients assessed for eligibility, 2653 were eligible for analysis. The dataflow is presented in Fig. 1.

Baseline characteristics for included patients are reported in Table 1. For characteristics of excluded data please see additional material [see Additional file 1].

\section{Main results}

\section{Stroke recognition}

For recognition of stroke and TIA, we found an overall sensitivity of $66.2 \%$ (95\% CI: 64.4\%-68.0 \%) and a PPV of $30.2 \%$ (95 \% CI: $29.1 \%-31.4 \%$ ), see Table 2 . Among the recognised cases, $79.3 \%$ were categorised as A.26.03 ("Lowered consciousness-paralysis, suspected stroke: Suddenly asymmetry in the face, lowered force in arms/legs, and/or verbal difficulties") and $20.7 \%$ as A.26.04 ("Lowered consciousness-paralysis, increasingly unclear/ listlesssuspected stroke").

\section{Factors associated with recognition of acute stroke}

Recognition varied among diagnoses (TIA: $73.8 \%$, AIS: $66.0 \%$, and $\mathrm{ICH}: 50.5 \%$; $P<0.0001)$. Variation in stroke recognition according to the time of day (Daytime (07-15): $67.8 \%$, Evening (15-23): $65.2 \%$, and Night - time (23-07): $60.2 \% ; P=0.0401$ ), and sex (Male: $68 \%$, Female: $64.3 \%$; $P=0.0406$ ) was detected. The multivariable logistic regression analyses were adjusted for sex, age, time of day and final diagnosis. This confirmed the results, however the effect of male sex on recognition diminished, see Table 3.

\section{Other analyses \\ Unrecognised and false positive cases}

Among the unrecognised cases, $46.7 \%$ were assigned the dispatch code "unclear problem" and $12.9 \%$ were assigned the dispatch code "unconscious (lifeless) adult". Please see additional material for detailed information about assigned dispatch codes for unrecognised cases [see Additional file 2].

Patients with suspected stroke by the EMD, but no record of stroke at hospital discharge, presented with a great variation of ICD-10 discharge diagnoses. The two most common were "Syncope and collapse" (3.2 \%) and "Other and unspecified symptoms and signs involving the nervous and musculoskeletal systems" (3.0\%). Please see additional material for all diagnoses registered for false positive cases [see Additional file 2].

The unrecognised cases were dispatched as a priority A response in $53.1 \%$ of cases and priority B in $46.6 \%$. Unrecognised cases with the "unclear problem" dispatch code assigned, were dispatched as a priority A response in $35.3 \%$ of cases and priority B response in $64.5 \%$ of cases, see Table 4 .

\section{Discussion}

\section{Key results}

The study showed an overall sensitivity of $66.2 \%$ and a PPV of $30.2 \%$ for recognition of AIS, ICH, and TIA during emergency calls. The multivariable logistic regression analyses showed that a final diagnose of TIA

Table 1 Characteristics for patients eligible for analysis

\begin{tabular}{llll}
\hline & Patients eligible for analysis $(n=2,653)$ & Recognised $(n=1,756)$ & Unrecognised $(n=897)$ \\
\hline $2012, n(\%)$ & $1,329(50.1)$ & $881(50.2)$ & $448(49.9)$ \\
Male, $n$ (\%) & $1,381(52.1)$ & $939(53.5)$ & $442(49.3)$ \\
Age, median (IQR) & 74 y (64-83) & 74 y (64-83) & 75 y (65-84) \\
Diurnal variation, $n$ (\%) & & & \\
-Day (07-15) & $1,575(59.4)$ & $1,067(60.8)$ & $396(44.2)$ \\
-Evening (15-23) & $812(30.6)$ & $529(30.1)$ & $378(42.1)$ \\
-Night (23-07) & $266(10.0)$ & $160(9.1)$ & $123(13.7)$ \\
Final ICD-10 diagnosis, $n(\%)$ & & & $181(20.2)$ \\
-DG459 & $692(26.1)$ & $511(29.1)$ & $155(17.3)$ \\
-Dl61 & $313(11.8)$ & $158(9.0)$ & $561(62.5)$ \\
-Dl63 & $1,648(62.1)$ & $1,087(61.9)$ & \\
\hline
\end{tabular}

Abbreviations: IQR inter quartile range, ICD-10 International Classification of Diseases, Tenth Revision 
Table 2 Recognition of stroke by emergency medical dispatchers

\begin{tabular}{|c|c|c|c|c|}
\hline & & \multicolumn{2}{|c|}{ Final diagnosis of stroke/TIA } & \multirow{2}{*}{$\begin{array}{l}\text { Total } \\
\text { N, \% (95 \% Cl) }\end{array}$} \\
\hline & & Yes & No & \\
\hline \multirow[t]{2}{*}{ Dispatch code for stroke/TIA } & Yes & 1,756 & 4,049 & 5,805 \\
\hline & No & 897 & 128,766 & 129.663 \\
\hline Total & & 2,653 & 132,815 & 135.468 \\
\hline Sensitivity & & & & $66.2 \%(64.4 \%-68.0 \%)$ \\
\hline Positive predictive value & & & & $30.2 \%(29.1 \%-31.4 \%)$ \\
\hline
\end{tabular}

Abbreviations: TIA transient ischemic attack, Stroke acute ischemic stroke or intracerebral haemorrhage, $\mathrm{Cl}$ Confidence Interval

and calls handled during the day were positively associated with recognition of acute stroke.

\section{Interpretation and generalisability Stroke recognition}

A sensitivity of $66.2 \%$ is high compared to other reported results ranging from $31 \%$ to $73.9 \%$ [20-24]. However a PPV of $30.2 \%$ is low compared other results ranging from $42 \%$ to $59.1 \%$ [21-23, 25]. The high sensitivity is likely the result of a profound reorganisation of the EMS Copenhagen during the past 5 years. This included, the introduction of EMDs with a medical profession (before it was police officers with no medical training) and the criteria-based dispatch tool Danish Index for Emergency Care. A recognition rate of $100 \%$ is not obtainable without an inappropriate amount of false positive cases; however there is still room for improvement.

Early recognition of TIA is of outermost importance as the risk of developing a stroke after a TIA is substantial, especially in the first coming days [26]; and up to $23 \%$ of AIS are preceded by a TIA [27]. Recognition of TIA provides an opportunity for early intervention and possible prevention of acute stroke.

Table 3 Results of multivariable logistic regression model identifying factors associated with recognition of stroke/TIA

\begin{tabular}{lll}
\hline Effects & Unadjusted OR $(95 \% \mathrm{Cl})$ & Adjusted OR $(95 \% \mathrm{Cl})^{\mathrm{a}}$ \\
\hline TIA vs. ICH & $2.77(2.10-3.66)$ & $2.70(2.04-3.57)$ \\
TIA vs. AIS & $1.45(1.20-1.78)$ & $1.43(1.15-1.78)$ \\
AIS vs. ICH & $1.90(1.49-2.43)$ & $1.89(1.48-2.41)$ \\
Day vs. Night & $1.39(1.07-1.82)$ & $1.38(1.02-1.87)$ \\
Day vs. Evening & $1.12(0.94-1.34)$ & $1.12(0.94-1.34)$ \\
Night vs. Evening & $0.81(0.61-1.07)$ & $0.81(0.61-1.09)$ \\
Age, y & $0.994(0.988-1.001)$ & $0.996(0.990-1.003)$ \\
Male & $1.18(1.01-1.39)$ & $1.17(0.99-1.38)$ \\
\hline
\end{tabular}

Abbreviations: $O R$ odds ratio, $\mathrm{Cl}$ confidence interval, $T I A$ transient ischemic attack, AIS acute ischemic stroke, $\mathrm{ICH}$ intracerebral haemorrhage

${ }^{a}$ Adjusted for sex, age, time of day and final diagnosis

\section{Factors associated with recognition}

The multivariable logistic regression analyses showed that calls during day-time, and final diagnoses of TIA and AIS were positively associated with recognition of acute stroke.

This finding is contrary to our expectations, as TIA was presumed more difficult to recognise as reported in another study [28]. This result is to our knowledge not reported elsewhere. The differences in recognition amongst final diagnoses, questions the otherwise general assumption that these conditions present similarly in patients. If any specific symptoms had a high predictive value for a specific pathology, it could facilitate the development of alternative prehospital scoring systems. Such could be of great value to the EMD, ambulance personnel, and the clinicians.

The diurnal variations in recognition of stroke could be explained by less awareness of stroke during the nights compared to day-time.

\section{Unrecognised patients}

Approximately $1 / 3$ of the stroke/TIA patients were not recognised during the emergency call. In $47 \%$ of the unrecognised cases, the EMD did not assign a priority A response, which may have delayed treatment for these patients, potentially impairing their outcome.

Among the unrecognised patients, the most present dispatch code was "unclear problem". The unclear problem category is a necessity in the dispatch system due to the complexity of emergency calls. For the ongoing revaluation of the Danish Index for Emergency Care, it is essential to stress that 421 patients, with subsequently

Table 4 Response priority of unrecognised cases in total and for unrecognised cases dispatched as "unclear problem"

\begin{tabular}{lll}
\hline Priority & Unrecognised in total, $n(\%)$ & $\begin{array}{l}\text { Unrecognised with "unclear } \\
\text { problem" dispatch code, } n(\%)\end{array}$ \\
\hline A & $476(53.1)$ & $148(35.3)$ \\
B & $418(46.6)$ & $270(64.5)$ \\
C & $3(0.3)$ & $1(0.2)$ \\
\hline
\end{tabular}

Priority A: Acute, Priority B: Urgent, Priority C: Scheduled 
confirmed stroke or TIA, were assigned the "unclear problem" category during a 2-year period.

Among the unrecognised patients with a dispatch code of "unclear problem", only $35.3 \%$ received a priority A response, compared to $53 \%$ among the unrecognised patients in total. This implies that when the EMD is in doubt, there is a tendency towards dispatching a less urgent response. This emphasises the importance of correct interpretation of symptoms.

\section{False positive cases}

With a PPV of $30.2 \%$, it is relevant to explore if certain conditions are often misinterpreted as a stroke by the EMD. The most common discharge diagnoses for false positive cases were "Syncope and collapse" and "Other and unspecified symptoms and signs involving the nervous and musculoskeletal systems". However, these diagnoses only represented 3.2 and $3.0 \%$ of false positive cases respectively, indicating that no specific conditions are systematically misinterpreted as stroke.

\section{Future aspects}

To decrease the prehospital delay, recognition by EMDs plays a major role and has the potential to reduce time from symptom onset to in-hospital evaluation; and thereby increase the proportion of patients receiving thrombolysis treatment.

Complete recognition is unobtainable, as the symptomatology is unspecific and differs among stroke patients. A higher recognition rate could though be obtained by encouraging the EMDs to suspect stroke in "not so obvious" cases. However this could result in over-dispatching, including an excessive number of dispatched priority A responses, resulting in under-prioritisation of other emergencies. Consequently, there would be an excessive risk of hazards and resource implications. In combination with the recognition rate, the PPV serves as an indicator of performance for international benchmarking between EMS organisations.

To achieve higher recognition rates without overdispatching, different approaches can be considered. It might be speculated that EMDs with a medical background provides the best foundation for medical dispatch. It is eminent to strive for a high level of recognition through feedback and further education, focused on the complex task of medical dispatching. During EMD training courses, the prospects of fast and precise handling of potential stroke patients must be emphasised. In addition, internal audit of calls is a great learning tool and can be incorporated in the daily supervision program at the EMDC.

The use of Danish Index for Emergency Care as a decision tool for emergency calls is recently validated [14]. However, constant revision of this dispatch tool is necessary to ensure high performance. In such revisions, the introduction of a standardised stroke scale for evaluation of stroke symptoms would be highly relevant. Furthermore, the exploration of the "unclear problem" dispatch code could hold a great potential, as nearly half of the unrecognised patients in this study were assigned this dispatch code.

The importance of medical dispatch and recognition of acute conditions during emergency calls is acknowledged in the medical literature, especially considering out-of-hospital cardiac arrest [11, 29, 30]. National, as well as international, benchmarking on stroke recognition could serve as a quality indicator, and potentially contribute to better outcome for stroke patients.

\section{Limitations and strengths}

Nearly $50 \%$ of the stroke/TIA patients were unmatchable with EMDC data. It is reasonable to assume that these patients went to the hospital without calling the emergency number. This assumption is supported in the literature [31].

\section{Conclusion}

This is an observational study of 2,653 emergency calls for patients suffering from AIS, ICH, or TIA, during a 2-year period from 2012 to 2014 in Copenhagen. The study reports an overall sensitivity of $66.2 \%$ and a PPV of $30.2 \%$ for recognition of AIS, ICH, and TIA during emergency calls. A final diagnose of TIA, compared to ICH, and calls handled during daytime were positively associated with recognition of acute stroke.

This study questions the otherwise general assumption that TIA, ICH, and AIS presents similarly in a clinical context, which could facilitate the development of supplementary prehospital scoring systems.

These results reports a potentially large group of stroke patients that are not recognised during the emergency calls, which could lead to prolonged time from symptom-onset to treatment and thereby worsening of outcome.

\section{Additional files}

\section{Additional file 1: Characteristics of total patient population and excluded patients within each step of exclusion (DOCX $19 \mathrm{~kb}$ )}

Additional file 2: Dispatch codes for unrecognised cases and ICD-10 discharge diagnoses for false positive cases (DOCX $15 \mathrm{~kb}$ )

\section{Abbreviations}

AIS, acute ischemic stroke; Cl, confidence intervals; DSR, The Danish Stroke Registry; EMD, Emergency Medical Dispatcher; EMDC, Emergency Medical Dispatch Centre; EMS, Emergency Medical Services; ICD-10, International Classification of Diseases, Tenth Revision; ICH, intracerebral haemorrhage; IQR, inter quartile range; NPR, The National Patient Registry; OR, odds ratio; PPV, positive predictive value; $\mathrm{SAH}$, subarachnoid haemorrhage; TIA, transient ischemic attack 


\section{Acknowledgements}

The research group is very grateful for the statistical assistance provided by Associate Professor Karl Bang Christensen from the Section of Biostatistics at the Department of Public Health, University of Copenhagen.

\section{Funding}

The author received an unrestricted grant from the Danish foundation "TrygFonden". TrygFonden was not involved in the design of the study, the collection, analysis, or interpretation of data, or the writing of the manuscript.

\section{Authors' contributions}

SV, TPM, HKI, HK, and FL planned and designed the study. SV and FL obtained funding for the study. HKI, HK, and FL supervised the execution of the study. SV and TPM managed the data. SV analysed the data. All authors contributed to the interpretation of the results. SV drafted the manuscript and all authors contributed substantially and critically to its revision. All authors read and approved the final version of the manuscript.

\section{Competing interests}

The Research Centre at the EMS Copenhagen receives unrestricted centre support from the Laerdal Foundation for Acute Medicine.

\section{Author details}

'Emergency Medical Services Copenhagen, University of Copenhagen, Telegrafvej 5, 2750 Ballerup, Denmark. ${ }^{2}$ Stroke Unit, Department of Neurology, Copenhagen University Hospital, Rigshospitalet-Glostrup, Nordre Ringvej 57, 2600 Glostrup, Denmark. ${ }^{3}$ Stroke Unit, Department of Neurology, Copenhagen University Hospital, Bispebjerg, Bispebjerg Bakke 23, 2400 Copenhagen, NV, Denmark.

\section{Received: 10 February 2016 Accepted: 16 June 2016} Published online: 07 July 2016

\section{References}

1. Schmitz ML, Simonsen CZ, Hundborg H, Christensen H, Ellemann K, Geisler K, et al. Acute ischemic stroke and long-term outcome after thrombolysis: nationwide propensity score-matched follow-up study. Stroke. 2014:45:3070-2.

2. Hacke W, Kaste M, Bluhmki E, Brozman M, Dávalos A, Guidetti D, et al Thrombolysis with alteplase 3 to $4.5 \mathrm{~h}$ after acute ischemic stroke. N Engl J Med. 2008;359:1317-29.

3. Wardlaw JM, Murray V, Berge E, del Zoppo GJ. Thrombolysis for acute ischaemic stroke. Cochrane Database Syst Rev. 2014;doi: 10.1002/14651858.

4. Emberson J, Lees KR, Lyden P, Blackwell L, Albers G, Bluhmki E, et al. Effect of treatment delay, age, and stroke severity on the effects of intravenous thrombolysis with alteplase for acute ischaemic stroke: a meta-analysis of individual patient data from randomised trials. Lancet. 2014;384:1929-35.

5. Saver JL, Fonarow GC, Smith EE, Reeves MJ, Grau-Sepulveda MV, Pan W, et al. Time to treatment with intravenous tissue plasminogen activator and outcome from acute ischemic stroke. JAMA. 2013;309:2480-8.

6. Danish Stroke Registry-2014 Annual Report, Thrombolysis supplement, https://www.sundhed.dk/content/cms/69/4669_trombolyse.pdf. Accessed 21 Dec 2015

7. Danish Stroke Registry-2014 Annual Report https://www.sundhed.dk/ content/cms/69/4669_dansk-apopleksi-register_aarsrapport_2014_ endeliqudgave2.pdf. Accessed 6 Nov 2015.

8. Barber PA, Zhang J, Demchuk AM, Hill MD, Buchan AM. Why are stroke patients excluded from TPA therapy? an analysis of patient eligibility. Neurology. 2001;56:1015-20.

9. Tong D, Reeves MJ, Hernandez AF, Zhao X, Olson DM, Fonarow GC, et al. Times from symptom onset to hospital arrival in the Get with the guidelines—stroke program 2002 to 2009: temporal trends and implications. Stroke. 2012:43:1912-7.

10. Lin CB, Peterson ED, Smith EE, Saver JL, Liang L, Xian Y, et al. Emergency medical service hospital prenotification is associated with improved evaluation and treatment of acute ischemic stroke. Circ Cardiovasc Qual Outcomes. 2012;5:514-22.

11. Acker JE, Pancioli AM, Crocco TJ, Eckstein MK, Jauch EC, Larrabee H, et al. Implementation strategies for emergency medical services within stroke systems of care a policy statement from the American heart association/
American stroke association expert panel on emergency medical services systems and the stroke council. Stroke. 2007;38:3097-115.

12. Jauch EC, Saver JL, Adams Jr HP, Bruno A, Connors JJB, Demaerschalk BM, et al. Guidelines for the early management of patients with acute ischemic stroke: a guideline for healthcare professionals from the American heart association/American stroke association. Stroke. 2013:44:870-947.

13. 2013 Referenceprogramme for Treatment of Patients with Stroke and TIA-Danish Stroke Society. http://www.dsfa.dk/wpcontent/uploads/ Nationale_retningslinier_trombolyse_2016_110216.pdf. Accessed 18 Nov 2015

14. Andersen MS, Johnsen SP, Sørensen JN, Jepsen SB, Hansen JB, Christensen EF. Implementing a nationwide criteria-based emergency medical dispatch system: a register-based follow-up study. Scand J Trauma Resusc Emerg Med. 2013; doi: 10.1186/1757-7241-21-53.

15. Kidwell CS, Saver JL, Schubert GB, Eckstein M, Starkman S. Design and retrospective analysis of the los Angeles prehospital stroke screen (lapss). Prehosp Emerg Care. 1998;2:267-73.

16. Simonsen S, Andresen M, Michelsen L, Viereck S, Lippert FK, Iversen H. Evaluation of pre-hospital transport time of stroke patients to thrombolytic treatment. Scand J Trauma Resusc Emerg Med. 2014;doi: 10.1186/s13049014-0065-z.

17. Schmidt M, Schmidt SAJ, Sandegaard JL, Ehrenstein V, Pedersen L, Sørensen HT. The Danish national patient registry: a review of content, data quality, and research potential. Clin Epidemiol. 2015;7:449.

18. Mainz J, Krog BR, Bjørnshave B, Bartels P. Nationwide continuous quality improvement using clinical indicators: the Danish national indicator project. Int J Qual Health Care. 2004;1:45-50. 16 Supp.

19. Pedersen CB. The Danish civil registration system. Scand J Public Health. 2011;39:22-5.

20. Rosamond WD, Evenson KR, Schroeder EB, Morris DL, Johnson A-M, Brice $J$ H. Calling emergency medical services for acute stroke: a study of 9-1-1 tapes. Prehosp Emerg Care. 2005;9:19-23.

21. Buck BH, Starkman S, Eckstein M, Kidwell CS, Haines J, Huang R, et al. Dispatcher recognition of stroke using the national academy medical priority dispatch system. Stroke. 2009:40:2027-30.

22. Deakin CD, Alasaad M, King P, Thompson F. Is ambulance telephone triage using advanced medical priority dispatch protocols able to identify patients with acute stroke correctly? Emerg Med J. 2009;26:442-5.

23. Krebes S, Ebinger M, Baumann AM, Kellner PA, Rozanski M, Doepp F, et al. Development and validation of a dispatcher identification algorithm for stroke emergencies. Stroke. 2012;43:776-81.

24. Handschu R, Poppe R, Rauss J, Neundörfer B, Erbguth F. Emergency calls in acute stroke. Stroke. 2003;34:1005-9.

25. Ramanujam P, Guluma KZ, Castillo EM, Chacon M, Jensen MB, Patel E, et al. Accuracy of stroke recognition by emergency medical dispatchers and paramedics_San Diego experience. Prehosp Emerg Care. 2008:12:307-13.

26. Giles MF, Rothwell PM. Risk of stroke early after transient ischaemic attack: a systematic review and meta-analysis. Lancet Neurol. 2007:6:1063-72.

27. Rothwell PM, Warlow CP. Timing of TIAs preceding stroke: time window for prevention is very short. Neurology. 2005:64:817-20.

28. Chenaitia H, Lefevre $\mathrm{O}, \mathrm{Ho}$ V, Squarcioni C, Pradel V, Fournier M, et al. Emergency medical service in the stroke chain of survival. Eur J Emerg Med. 2013;20:39-44.

29. Berdowski J, Beekhuis F, Zwinderman AH, Tijssen JG, Koster RW. Importance of the first link: description and recognition of an out-of-hospital cardiac arrest in an emergency call. Circulation. 2009;119:2096-102.

30. Ornato JP. Science of emergency medical dispatch. Circulation. 2009;119: 2023-5.

31. Mohammad YM. Mode of arrival to the emergency department of stroke patients in the United States. J Vasc Interv Neurol. 2008;1:83-6. 